\title{
Magnetic Fringe-Field Control of Electronic Transport in an Organic Film
}

\author{
Fujian Wang, ${ }^{1}$ Ferran Macià, ${ }^{2}$ Markus Wohlgenannt, ${ }^{1}$ Andrew D. Kent, ${ }^{2}$ and Michael E. Flatté ${ }^{1}$ \\ ${ }^{1}$ Department of Physics and Astronomy and Optical Science and Technology Center, University of Iowa, \\ Iowa City, Iowa 52242, USA \\ ${ }^{2}$ Department of Physics, New York University, 4 Washington Place, New York, New York 10003, USA
}

(Received 27 March 2012; published 27 June 2012)

\begin{abstract}
Random, spatially uncorrelated nuclear-hyperfine fields in organic materials dramatically affect electronic transport properties such as electrical conductivity, photoconductivity, and electroluminescence. The influence of these nuclear-hyperfine fields can be overwhelmed by a uniform externally applied magnetic field, even at room temperature where the thermodynamic influences of the resulting nuclear and electronic Zeeman splittings are negligible. As a result, even in applied magnetic fields as small as $10 \mathrm{mT}$, the kinetics of exciton formation, bipolaron formation, and single-carrier hopping are all modified at room temperature, leading to changes in transport properties in excess of $10 \%$ in many materials. Here, we demonstrate a new method of controlling the electrical conductivity of an organic film at room temperature, using the spatially varying magnetic fringe fields of a magnetically unsaturated ferromagnet. (The fringe field is the magnetic field emanating from a ferromagnet, associated with magnetic dipole interactions or, equivalently, the divergence of the magnetization within and at the surfaces of the ferromagnet.) The ferromagnet's fringe fields might act as a substitute for either the applied magnetic field or the inhomogeneous hyperfine field. The size of the effect, the magnetic-field dependence, and hysteretic properties rule out a model where the fringe fields from the ferromagnet provide a local magnetic field that changes the electronic transport properties through the hyperfine field, and show that our effects originate from electrical transport through the inhomogeneous fringe fields coming from the ferromagnet. Surprisingly, these inhomogeneous fringe fields vary over length scales roughly 2 orders of magnitude larger than the hopping length in the organic materials, challenging the fundamental models of magnetoresistance in organic layers which require the correlation length of the inhomogeneous field to correspond roughly to the hopping length.
\end{abstract}

DOI: 10.1103/PhysRevX.2.021013

\section{INTRODUCTION}

Most devices that rely on electron-spin dynamics to function (spintronics) use a change in the relative magnetizations of two magnetic electrodes to control the flow of electronic current through a nonmagnetic material [1-3], such as by spin-selective scattering (as in current-in-plane giant magnetoresistance) [4,5] or by spin-injection (as in current-perpendicular-to-plane giant magnetoresistance) [6]. For the simpler geometry of a single magnetic electrode, spin-orbit effects modify the injection process of carriers at the interface between magnetic and nonmagnetic materials through differences in the tunneling matrix elements (called tunneling anisotropic magnetoresistance, or TAMR) [7]. The resistance and related electrical properties of nonmagnetic organic materials are known, however, to change by several percent at room temperature even in very small magnetic fields (about $10 \mathrm{mT}$ ), due to the interplay between the applied magnetic field and the random, spatially uncorrelated nuclear-hyperfine fields

Published by the American Physical Society under the terms of the Creative Commons Attribution 3.0 License. Further distribution of this work must maintain attribution to the author(s) and the published article's title, journal citation, and DOI.
Subject Areas: Magnetism, Semiconductor Physics

[8-15]. Thus, the presence of a single, electrically isolated magnetic layer might be thought to be sufficient to similarly alter the resistance of an organic device at room temperature.

Here, we demonstrate such an effect. Remarkably, even in samples with no electrical contact to a magnetic layer, and only a single magnetic layer, magnetoresistance features similar to those reported for organic spin valves can be observed. Our devices, which do not rely on spin injection [16,17], tunneling anisotropic magnetoresistance [7], or spin-valve behavior [18-22], may provide a simple approach to integrating magnetic metals and organics for hybrid spintronic devices.

The magnetoresistive effects from a single magnetic electrode that we find are substantial (several percent) at room temperature can be engineered by controlling the domain structure of the magnetic electrode, and are not sensitive to injection at the magnetic-metal-organic interface. We have demonstrated the same effect in devices in which the ferromagnetic region is isolated from the current path, which completely eliminates spin injection, spinvalve behavior, and TAMR as a source of the magnetoresistive effects, even though the magnetoresistive curves strongly resemble those expected from spin valves. Organic materials are known to experience a large 
magnetoresistive effect at room temperature due to small applied fields (about $10 \mathrm{mT}$ ), which can overwhelm random nuclear-hyperfine fields, but, as we show below, this organic magnetoresistive (OMAR) effect (modified by fringe fields from the magnetic electrode) [23] cannot explain our data, because the size of the effect we observe is far too large, is seen in a very different magnetic-field range, has the wrong trend with distance between organic layer and electrode, and has the wrong sign of the hysteresis loop.

\section{MAGNETIC-LAYER PROPERTIES AND CHARACTERIZATION}

The strength and spatial-correlation length of magnetic fringe fields induced by a ferromagnet depend sensitively on the distance from the ferromagnet to the nonmagnetic organic film as well as the magnetic domain structure [24]. For a magnetic layer with a well-defined magnetic anisotropy, this domain structure is highly anisotropic and hysteretic. It can also be controlled by engineering the magnetic anisotropy and coercive fields of magnetic media
$[24,25]$. A film of alternating Co and Pt layers with perpendicular magnetic anisotropy (PMA) is the bottom electrode and the source of magnetic fringe fields in many of our devices, but similar results are obtained if the film is isolated from the current path of the device. This magnetic electrode with perpendicular magnetic anisotropy, deposited on a $\mathrm{Si} / \mathrm{SiO}_{2}$ substrate, can be magnetized by large perpendicular magnetic fields and demagnetized by large in-plane magnetic fields; it is terminated with (nonmagnetic) Pt.

It is a characteristic of smooth, saturated, perpendicularly magnetized films that they produce no fringe fields above the films far from the edges; fringe fields above the film originate from the presence of domains in the unsaturated state, or from thickness variations in the film. The properties of the ferromagnetic electrodes have been characterized in detail using a variety of complementary techniques. The polar magneto-optic Kerr effect (MOKE) has been used to measure the electrode's hysteresis loop and to correlate the loop with magnetotransport properties of organic semi-spin-valve devices. We use vibrating-sample magnetometry (VSM) to determine the electrodes' magnetization versus field. The electrode is also studied with

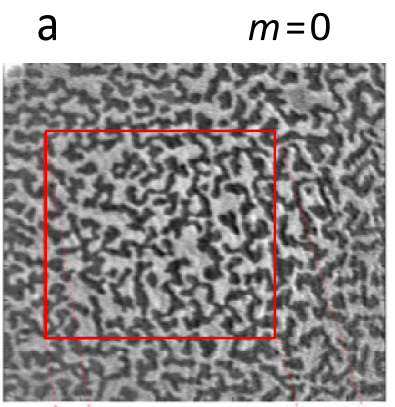

b
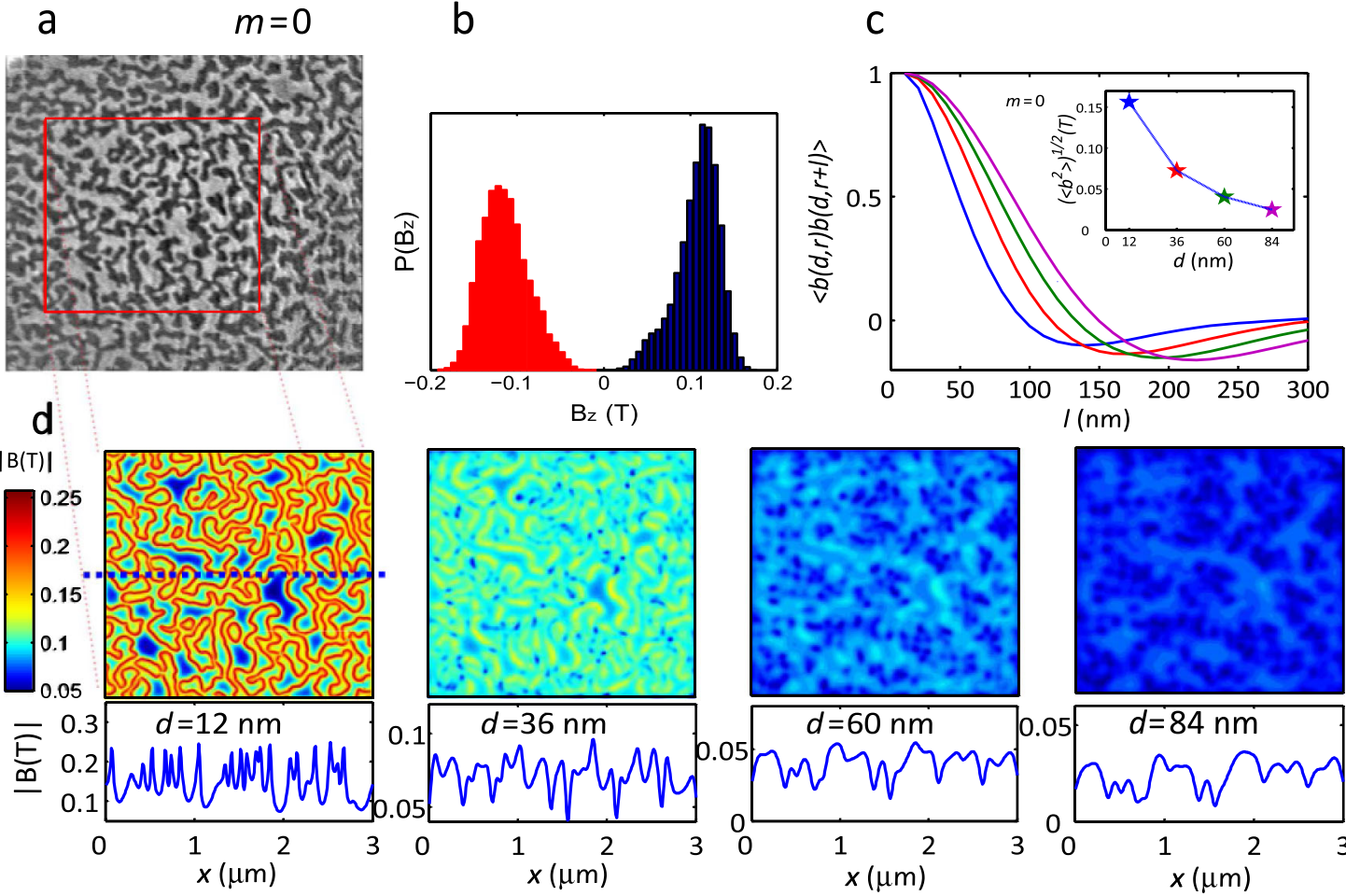

FIG. 1. Fringe fields from the magnetic electrode. (a) X-ray microscopy image of the magnetic electrode at the coercive field (corresponding to $m=0$ ). The signal is due to the dichroic absorption of circularly polarized x rays at the $L_{3}$ Co absorption edge incident normal to the film surface. The image is $5 \times 5 \mu \mathrm{m}^{2}$. The fringe fields were calculated from these images using OOMMF code [26]. (b) Distribution of perpendicular magnetic fringe fields, $B_{z}$, calculated from (a) at a distance of $12 \mathrm{~nm}$ from the magnetic electrode. (c) Normalized in-plane correlation function of the fringe fields at different distances from the electrode, $12 \mathrm{~nm}$ blue, $36 \mathrm{~nm}$ red, $60 \mathrm{~nm}$ green, and $84 \mathrm{~nm}$ purple. The inset shows the rms value for the fringe field vs distance from the electrode. (d) Magnitude of the fringe field in a $3 \times 3-\mu \mathrm{m}^{2}$ area is plotted using a color scale at four different distances from the electrode. The lower panels of (d) correspond to the fringe field as a function of position at $y=1.5 \mu \mathrm{m}$, a profile through the center of the corresponding color images. 
ferromagnetic resonance (FMR) spectroscopy, which provides a direct measurement of the electrode's perpendicular magnetic anisotropy. Finally, we use transmission x-ray microscopy (TXM) based on the x-ray magnetic circular dichroism (XMCD) effect to determine the electrodes' microscopic magnetic domain structure as a function of the applied perpendicular field. This detailed characterization is essential for determining reliably the nature of the fringe fields located above the ferromagnetic layer and within the organic device. In this paper, we focus on the TXM results; the other characterization results are shown in Appendix A.

We characterize the domain structure of the magnetic layer as a function of applied field using TXM and normalincidence XMCD [27] of the Co $L_{3}$ absorption edge. X-ray measurements were performed at the Advanced Light Source at the Lawrence Berkeley National Laboratory. Images were taken with an XM-1 zone-plate microscope at beam line 6.1.2. XMCD measurements [Fig. 1(a)] were conducted at the coercive field of the electrode (magnetization $m \approx 0$ ) and show "up" (white) and "down" (black) magnetized domains of approximately $200 \mathrm{~nm}$ in a labyrinth pattern. (See Appendix A for additional images at different applied fields, and the Supplemental Material for videos of the domain dynamics [28].) These images have been used to determine the fringe magnetic fields at different distances above the ferromagnetic layer; the distances were calculated from the magnetization and the geometry using object-oriented micromagnetic framework (OOMMF) code [26].

The distribution of fringe fields perpendicular to the film is shown in Fig. 1(b), showing a range between -0.2 and $0.2 \mathrm{~T}$, peaked near $\pm 0.1 \mathrm{~T}$. Figure 1(c) plots the lateral correlation function of the fringe fields. This figure shows that the correlation length of the fringe fields increases with increasing distance from the surface, while the root-mean-square (rms) amplitudes of the fields decay rapidly with distance [inset, Fig. 1(c)]. Higher Fourier components of the fringe fields decay more rapidly with distance from the electrode surface, leading to a smoother variation in the fringe field and a larger field correlation length. The four panels of Fig. 1(d) correspond to the fringe fields at four different distances above the electrode, calculated from image Fig. 1(a) using micromagnetics. Atomic-force-microscopy measurements on similar films show a variation in the magnetic layer thickness of no more than $2 \mathrm{~nm}$ on the micron scale. Lateral thickness variations on the 10 -nm length scale would be most effective at creating a large fringe-field variation $30 \mathrm{~nm}$ above the film. Assuming a 1-nm thickness variation on this lateral scale, we find a fringe field from magnetostatic calculations of about $1 \mathrm{mT}$, which is much smaller than the relevant field scale. Thus, layer thickness variations and the resulting fringe fields will be neglected in our analysis.

\section{SEMI-SPIN-VALVE DEVICE FABRICATION AND PROPERTIES}

The organic semiconductor device, called a semi-spin valve [Fig. 2(a)], consists of a ferromagnetic layer and (sometimes) an electrically isolating layer, followed by a nonmagnetic metal, a hole-injecting layer, an organic semiconductor, and a top electrode. The ferromagnetic layer is a Co|Pt multilayered film. (The multilayer consists of 30 repetitions of a 3.0-nm Co|0.5-nm Pt unit cell, terminated with $5 \mathrm{~nm}$ of Pt.) The film is deposited using electron-beam evaporation in ultrahigh vacuum on oxidized $\mathrm{Si}$ wafers for studying devices and $\mathrm{Si}$-supported $\mathrm{Si}_{3} \mathrm{~N}_{4}$ windows for the magnetic-domain imaging studies using an x-ray transmission microscope. Some devices have $\mathrm{SiO}_{2}$ deposited on them subsequently, followed by $12 \mathrm{~nm}$ of Pt to electrically isolate the ferromagnetic layer. Optical lithography is then used to define the bottomelectrode geometry. A hole-injecting layer follows, either sputtered indium tin oxide (ITO) or the conducting polymer poly(3,4-ethylenedioxythiophene) poly(styrenesulfonate) (PEDOT PSS) deposited by spin coating from an aqueous suspension. (The suspension comes from H.C. Starck, CLEVIO P VP AI 4083.) A 30-nm-thick film of the organic semiconductor tris(8-hydroxyquinoline aluminum $\left(\mathrm{Alq}_{3}\right.$, sublimed grade, from H.W. Sands Corporation) is deposited by thermal evaporation in high vacuum and at room temperature. A 10-nm Ca layer is used to take advantage of the favorable work function of $\mathrm{Ca}$; it is covered by a 40-nm capping layer of $\mathrm{Al}$. These layers are deposited by electron-beam evaporation at room temperature through a metal stencil to obtain a cross-point device geometry of dimension $500 \times 500 \mu \mathrm{m}$. The contributions to the device resistance (and magnetoresistance) of the PEDOT or ITO conducting layer and the Ca electrode are negligible, since they are metals, whereas $\mathrm{Alq}_{3}$ is an intrinsic semiconductor.

Both PEDOT and ITO devices produced very similar results and all measurements were at room temperature. $\mathrm{Alq}_{3}$ also has a particularly large organic magnetoresistive effect (see Ref. [29] and references therein), and the magnetoresistive response here occurs inside the $\mathrm{Alq}_{3}$ layer. In summary, the current path in our devices is bottom electrode $\rightarrow$ PEDOT or ITO $\rightarrow \mathrm{Alq}_{3} \rightarrow \mathrm{Ca} \rightarrow$ Al. The PEDOT or ITO hole-injecting layer also serves as a spacer layer of variable thickness to separate the magnetoresistive material, $\mathrm{Alq}_{3}$, from the magnetic layer that is the source of the magnetic fringe fields. In order to conclusively eliminate effects associated with spin-polarized transport or injection (including TAMR), in some devices the ferromagnetic layer was covered with a dielectric and then capped with $12 \mathrm{~nm}$ of Pt. Similar magnetoresistive curves have been obtained in these devices, demonstrating that the effect persists even when the ferromagnetic layer is excluded from the current path. 

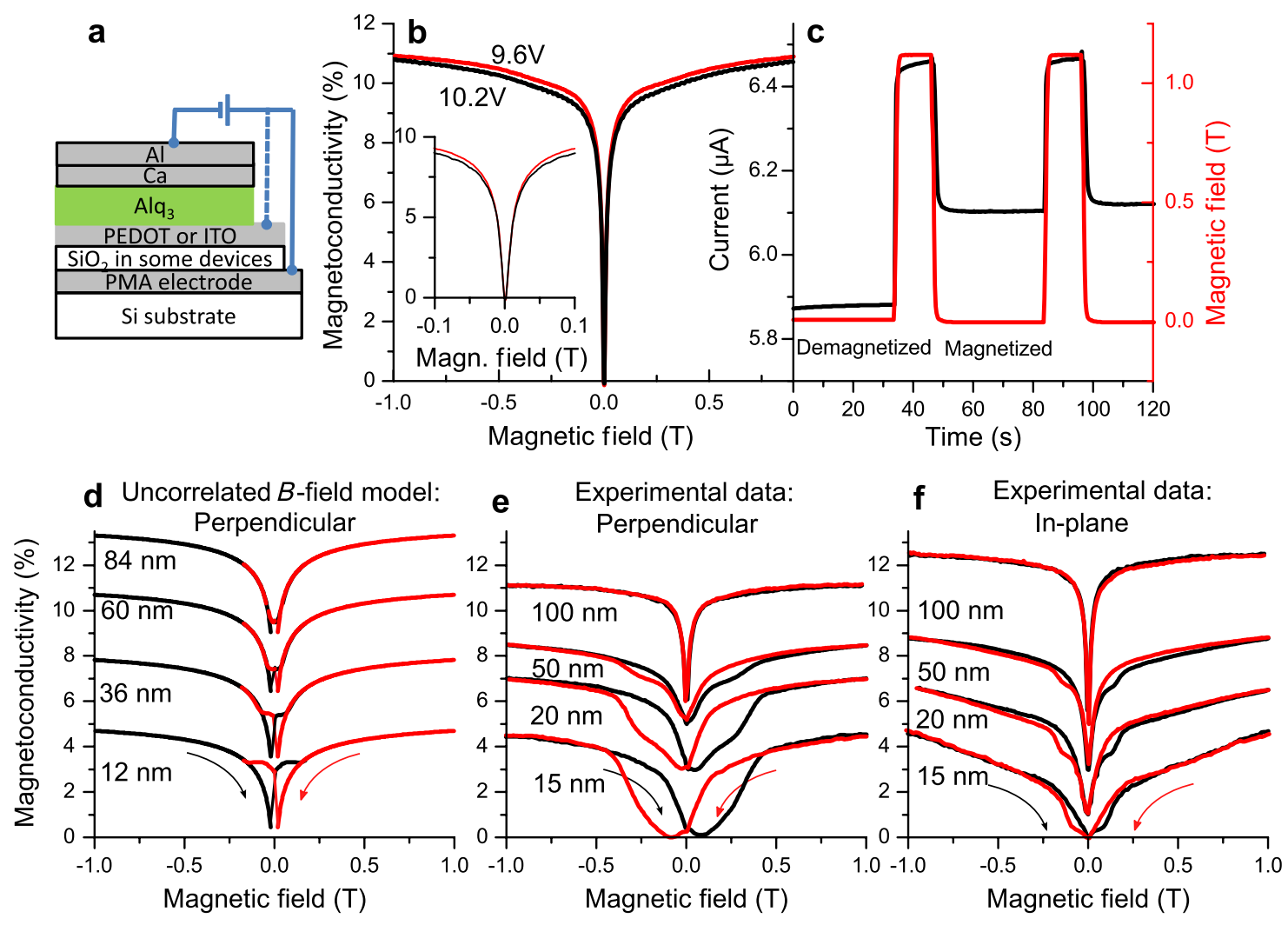

FIG. 2. Magnetoresistance in organic semiconductor devices. (a) Device schematic. (b) Organic magnetoconductivity caused by random hyperfine fields in a device using two nonmagnetic electrodes. (c) Hysteretic conductance in an organic semiconductor semispin valve with a bottom ferromagnetic electrode. Initially, the electrode is in a demagnetized state. At time 35 s, a magnetizing magnetic field is applied (red curve). Even after the magnetizing magnetic field is removed, the device conductivity (black curve) remains several percent above its original value. (d) Expectation for devices based on an uncorrelated $B$-field model, described in Appendix B, which considers the fringe fields to modify the local conductivity through the nonmagnetic organic magnetoresistance in (b). (e),(f) Dependence of the magnetoconductivity on the thickness of the PEDOT spacer layer between the ferromagnetic electrode and the magnetoresistive organic semiconductor for (e) perpendicular and (f) in-plane applied magnetic fields, respectively. The black curves are for sweeps from large negative fields to large positive fields, and the red curves for the return sweep. The numbers assigned specify the PEDOT spacer-layer thickness. (The data have been offset along the $y$ axis for added clarity.) The applied voltage biases are $4.5,7,7.2$, and $7.5 \mathrm{~V}$ for the $15,20,50$, and $100 \mathrm{~nm}$ devices, respectively. At small spacer-layer thickness, the magnetoconductivity is dominated by the hysteretic fringe-field-induced magnetoconductance, whereas for large spacer-layer thickness, we recover the hyperfine-induced magnetoconductance shown in (b).

\section{A. Magnetoresistance in semi-spin valves}

Figure 2(b) shows a typical magnetoconductivity trace for an organic device, fabricated without a ferromagnetic layer (using a bottom electrode of ITO), whose magnetoconductivity is caused by random hyperfine fields. The magnetoconductivity (or magnetoresistance) is defined here to be the difference between the conductivity measured at finite magnetic field and that measured at zero magnetic field, normalized by the conductivity (or resistance) at zero magnetic field. This device will serve as a reference for results on fringe-field-induced magnetoconductivity. Figure 2(b) shows that the hyperfine-induced magnetoconductivity response has a magnitude of several percent in our $\mathrm{Alq}_{3}$ devices. The effect reaches its limiting value for fields in excess of $0.1 \mathrm{~T}$, is nonhysteretic, and has a full width at half maximum of approximately $20 \mathrm{mT}$ (see inset). The effect is also independent of the direction of the applied magnetic field, and nearly independent of the thickness of the $\mathrm{Alq}_{3}$ layer. Magnetoresistance measurements (MR) were performed in a closed-cycle He cryostat positioned between the poles of an electromagnet. The measurements reported here are all at room temperature and were performed using a Keithley 2400 source meter.

The organic semi-spin valve shows an initially low device conductivity at zero magnetic field. Conductivity increases by several percent as the initially demagnetized ferromagnetic layer is magnetized when we apply a large field out of plane [Fig. 2(c)]. The conductivity at zero magnetic field decreases when the magnetizing field is removed, but it remains significantly above the level for the demagnetized device. This hysteretic behavior is in contrast to the hyperfine-induced magnetoconductivity of the reference device and clearly demonstrates an effect of 
the magnetic electrode on the device conductivity. (See Appendix A.)

We next consider a model, referred to as an "uncorrelated $B$-field model," in which the local fringe field from the ferromagnet (shown in Fig. 1) changes the local conductivity of the organic layer through ordinary organic magnetoresistance. The local conductivities are then averaged to produce the curves in Fig. 2(d). The model considers the organic semiconductor's conductivity to be a function of the local field $[H(\mathbf{x})]$ that it experiences, and the overall conductance is due to channels averaged in parallel. The conductance of a channel is $P(H) C(H)$, where $P$ is the fringe-field probability distribution and $C(H)$ is the conductivity of a channel [taken from Fig. 2(b)] for a given local field $H=h+H_{0}$. ( $h$ are the fringe fields, and $H_{0}$ is the externally applied field.) The net conductance is then just the sum of the conductances of each channel weighted by the number of such channels (i.e., the sum of $C(H) P(H)$ ). In zero applied field, $H_{0}=0 \mathrm{~T}$, and $H(r)$ is due entirely to the fringe fields.

Figures 2(e) and 2(f) show experimental data for perpendicular and in-plane applied magnetic fields, respectively, and show the magnetoconductivity as the applied magnetic field is swept smoothly from large negative to large positive fields (black lines) and back (red lines). For both perpendicular [Fig. 2(e)] and inplane [Fig. 2(f)] applied-field configurations, magnetoresistive effects of several percent are achieved for large fields, but the functional dependence of the magnetoconductivity on the applied field is anisotropic. The curves in Fig. 2(d) are displaced by $3 \%$ for improved visibility. The curves in Figs. 2(e) and 2(f) are also displaced; for these, the actual values of the curves at zero magnetic field are all $0 \%$. Measurements of the magnetic-domain structure from Fig. 1 are for a perpendicular magnetic field, which allows direct correlation of the domain structure with the magnetoconductivity, such as through the uncorrelated $B$-field model. For this reason, we do not further discuss the in-plane magneticfield results of Fig. 2(f).

Figures 2(e) and 2(f) contain several data sets that show that the hysteretic response of the semi-spin-valve effect gradually decreases with increasing PEDOT spacer-layer thickness (i.e., the distance between the magnetic electrode and the magnetoresistive organic semiconductor) and vanishes for a spacer-layer thickness of $100 \mathrm{~nm}$, whereas the uncorrelated $B$-field model in Fig. 2(d) shows that the effect for positive fields at positive sweep (black curves) and negative fields during a negative sweep (red curves) actually increases with increasing separation. Furthermore, the sign of the hysteresis seen in the data in Fig. 2(e) is opposite that seen in the model in Fig. 2(d). Finally, note that the range of fields for which hysteresis is seen in the model does not exceed $0.1 \mathrm{~T}$, whereas, in the data, the range of fields with visible hysteresis approaches $0.5 \mathrm{~T}$ for the closest organic layer. Thus, in trend, magnitude, and sign, the uncorrelated $B$-field model fails to explain the data.

Figure 3 shows magnetoconductivity curves and their correlation with magnetic hysteresis loops for two devices with ferromagnetic electrodes that have different PMA. Although the two electrodes have the same nominal layer stack, they differ slightly in magnetic properties due to small, unintentional variations in the fabrication process. (The magnetic properties of these two electrodes are discussed in Appendix A.) Hysteresis loops were measured
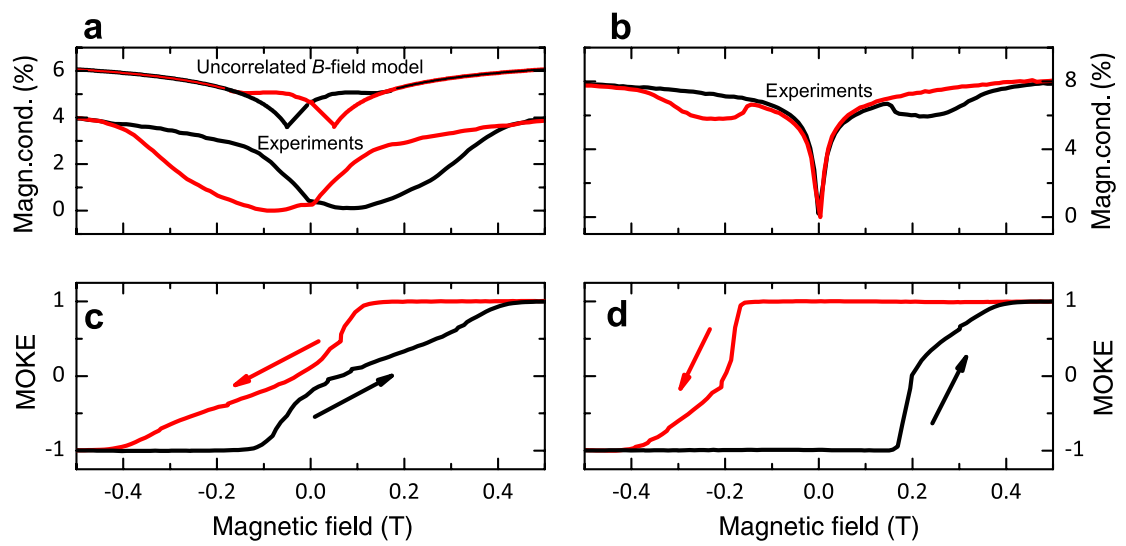

FIG. 3. Correlation between magnetoconductivity and magnetic switching for two different ferromagnetic bottom electrodes. (a),(b) show magnetoconductivity in semi-spin-valve devices; (c),(d) show the magneto-optic Kerr-effect (MOKE) measurements of the magnetic electrodes. The experiments were performed using a perpendicular applied magnetic field. The devices in (a),(b) used a 15- and 20-nm PEDOT spacer layer, respectively. The magnetic electrodes are nominally identical but have different magnetic properties due to variations in the fabrication process. This figure shows that the hysteretic magnetoresistance response correlates well with the magnetic state of the bottom electrode as determined by the MOKE data. Hysteretic magnetoconductivity is observed only in the regime where the ferromagnet is unsaturated and emits magnetic fringe fields that rapidly vary spatially. The uncorrelated $B$-field model in (a) disagrees with the measurement. 
using the magneto-optic Kerr-effect (MOKE) technique. The electrode shown in Fig. 3(a) has a smaller coercive field $(0.1 \mathrm{~T})$ than that in Fig. 3(b) $(0.2 \mathrm{~T})$. For both electrodes, the magnetoconductivity changes are largest at the coercive field, and the conductivity is suppressed between the nucleation field and the saturation field of the ferromagnetic electrode. We note that a saturated magnetic electrode does not generate fringe fields and therefore does not change the typical hyperfine-induced OMAR magnetoconductance near zero field.

The uncorrelated $B$-field model, shown in Fig. 3(a), disagrees both with the sign of the hysteresis and with the magnetic-field range of the hysteresis loop, showing that this model fails to describe the data. In the device with the electrode that has the larger coercivity [Fig. 3(b)], the effect of the hyperfine-induced magnetoconductivity variations (near zero applied field) occurs in a separate applied magnetic-field range from the variations induced by the ferromagnetic electrode (near the coercive field). These data show that the hysteretic magnetoresistance of the organic layer is directly associated with hysteretic magnetization of the ferromagnetic electrode, and that the conductance of the semi-spin valve is suppressed when there are magnetic domains in the ferromagnetic electrode.

\section{B. Magnetoresistance in PEDOT-only devices}

Our analysis relies on the notion that the magnetoconductivity response occurs in the $\mathrm{Alq}_{3}$ layer, and that any magnetoconductivity of the PEDOT makes a negligible contribution to the measured response. Here, we provide the experimental data to substantiate this claim. For this purpose, we conducted magnetoconductivity measurements in devices like those used in Sec. III A, but without the $\mathrm{Alq}_{3}$ layer. The device structure therefore was as follows: bottom ferromagnetic electrode, PEDOT $(40 \mathrm{~nm})$, $\mathrm{Ca}$, and Al. Figure 4 shows the magnetoconductivity as the applied magnetic field is swept smoothly from large negative to large positive fields (black lines); the return sweep is shown in red. This figure, which is analogous to Fig. 3, shows that, in PEDOT, magnetoresistive effects of about $0.4 \%$ are achieved for large fields, roughly 10 times smaller than the effects in a comparable device made with $\mathrm{Alq}_{3}$. In addition, it is important to understand that the resistance of the PEDOT layer, which is metallic, is several orders of magnitude smaller than the resistance of the $\mathrm{Alq}_{3}$ layer, which is an intrinsic semiconductor. Therefore, the overall device resistance (and therefore the device's magnetoconductance) in the PEDOT/ $\mathrm{Alq}_{3}$ device is strongly dominated by that of the $\mathrm{Alq}_{3}$ layer.

\section{Magnetoresistance in devices where the synthetic metal electrode is replaced by an inorganic metal}

Figure 5 shows the magnetoconductance measured in a control device in which the synthetic metal (PEDOT)

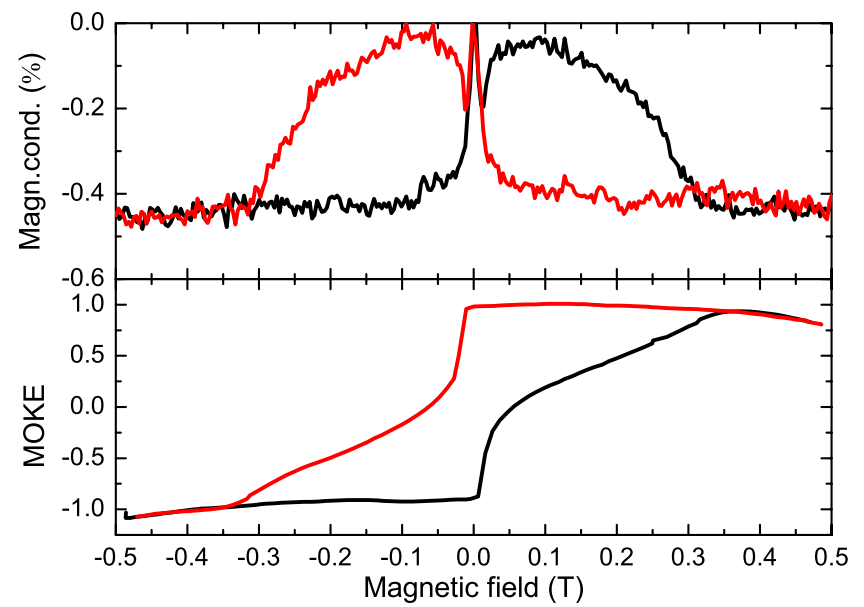

FIG. 4. Magnetoconductivity of a PEDOT-only semi-spin valve. The top panel shows the magnetoconductivity in semi-spin-valve control devices without the $\mathrm{Alq}_{3}$ layer. The device structure consists of a ferromagnetic bottom electrode, the 40-nm PEDOT spacer layer, $\mathrm{Ca}$, and Al. The bottom panel shows the magneto-optic Kerr-effect (MOKE) measurements of the magnetic electrode. The experiments were performed in a perpendicular applied magnetic field.

bottom electrode is replaced with an inorganic metal, indium tin oxide. ITO is a commonly employed electrode material for organic light-emitting diodes. In our device, we achieve a similar magnetoconductance effect as that reported for a comparable device with a PEDOT electrode [see Fig. 3(b)]. This result demonstrates that the observed magnetoresistance occurs in the organic semiconducting $\left(\mathrm{Alq}_{3}\right)$ layer and is largely independent of the exact nature of the bottom electrode.

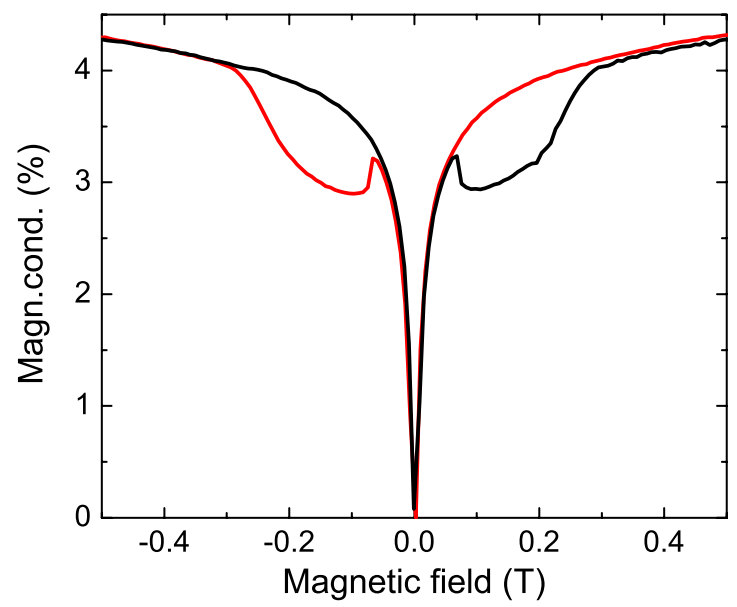

FIG. 5. Magnetoconductivity of devices with an inorganic metallic electrode. The device structure consists of a ferromagnetic bottom electrode, a 38-nm ITO layer, a 40-nm Alq ${ }_{3}$, a Ca layer, and an Al layer. The domain structure of this ferromagnetic electrode is similar to that employed in the device reported in Fig. 3(b). 


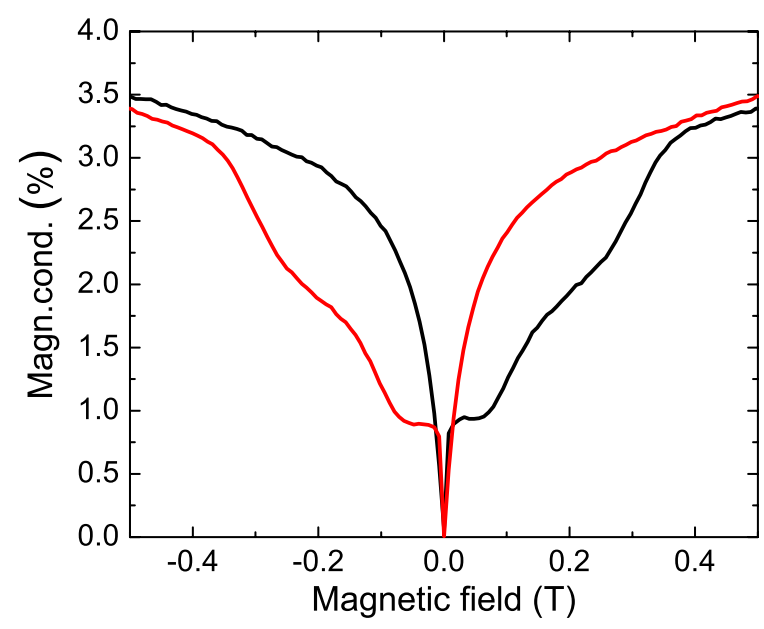

FIG. 6. Magnetoconductivity of devices with an electrically isolated ferromagnetic layer. The device structure consists of a ferromagnetic bottom electrode, a $12-\mathrm{nm} \mathrm{SiO}_{2}$ layer, a 13-nm Pt layer, a 20-nm PEDOT layer, a 40-nm $\mathrm{Alq}_{3}$, a Ca layer, and an Al layer. The domain structure of this ferromagnetic electrode is similar to that employed in the device reported in Fig. 3(a).

\section{Magnetoresistance of devices with an electrically isolated ferromagnetic layer}

Our analysis of the data relies on the claim that our semi-spin-valve effect is not caused by polarized spin injection but by the fringe-field distribution inside the organic semiconducting layer. Here, we prove this claim by a control experiment in which the organic magnetoresistive device is electrically isolated from the ferromagnetic film. A thin dielectric has been deposited on the ferromagnetic film and capped with a 12-nm Pt layer. The current path is therefore entirely outside the ferromagnetic thin film (i.e., in the Pt layer), and spin injection cannot occur. Figure 6 shows the magnetoconductivity as the applied magnetic field is swept smoothly from large negative to large positive fields (black lines); the return sweep is shown in red. This figure shows that the magnetoconductivity effect has a magnitude of about 3.5\% and exhibits a similar line-shape as that shown in Fig. 3(a). This experiment proves that our semi-spin-valve effect is not caused by spin injection.

\section{MECHANISM FOR FRINGE-FIELD MAGNETORESISTANCE}

We now summarize the novel magnetic fringe-field effect and its fundamental differences from the nuclearhyperfine organic-magnetoresistive effect. Figure 7 describes the qualitative model for the nuclear-hyperfine effect on organic magnetoresistance, as well as a typical distribution of magnetic fringe fields from a ferromagnetic electrode, and our proposed model for this new magnetic fringe-field effect on organic magnetoresistance. Figures 7(a) and 7(b) show schematically a low-field and high-field scenario for nuclear-hyperfine organic magnetoresistance. At low fields, the local spin-quantization axes are set by the (random) nuclear-hyperfine fields, whereas, at high fields, the local spin-quantization axes are set by the uniform applied magnetic field. Transport through the organic film occurs by a sequence of hops along a path connecting the top electrode to the bottom electrode (Fig. 7, brown lines), and the rate of transport is dramatically affected by variations in the local spin-quantization axis along the path, as found in experimental and theoretical studies [8-11,14]. If the source of that inhomogeneous field is a nuclear-hyperfine random field, as shown in Fig. 7(a), then the field is intrinsic, random, and spatially uncorrelated. An applied magnetic field $B$ that exceeds this random hyperfine field $B_{\mathrm{HF}}$, as shown in Fig. 7(b), changes the resistivity uniformly through the film; thus, the magnetoresistance is independent of distance from the nonmagnetic electrode as well as of the applied magnetic-field angle, producing the magnetoresistive curve shown in Fig. 7(d).

\section{A. Mechanism by which a fringe field replaces an applied field}

The spatially inhomogeneous magnetic field from the magnetic electrode cannot drive a substantial magnetoresistance via the same mechanism as shown in Fig. 7(a) and 7(b) because the magnitude of the spatially inhomogeneous magnetic field is greater than $B_{\mathrm{HF}}$ almost everywhere. When the applied field exceeds $0.2 \mathrm{~T}$, it is larger than the maximum perpendicular magnetic fringe field from the magnetic electrode [see Fig. 1(b)]. In this case, the magnitude of the total magnetic field (fringe plus applied) exceeds $B_{\mathrm{HF}}$ everywhere, which is why the uncorrelated $B$-field model in Fig. 3 does not show hysteresis beyond about $0.2 \mathrm{~T}$. In the uncorrelated $B$-field model, when domains are present [positive fields for up-sweep (black) and negative fields for down-sweep (red) in Fig. 2(d)], one would thus see a similar magnetoresistance to that seen in a large magnetic field in a nonmagnetic device, whereas, in the data [Fig. 2(e)] when domains are present, one sees magnetoresistance similar to that seen in a small magnetic field in a nonmagnetic device. The hysteretic magnetoresistance data, moreover, do not reach a limiting value until more than $0.4 \mathrm{~T}$, which suggests that the variance of the inhomogeneous field governing electronic transport has a scale of $0.2 \mathrm{~T}$ (which is the scale of the inhomogeneous fringe field).

In addition to the failure to explain the magnetic-field range where the effect is observed, the uncorrelated $B$-field model cannot explain the strength of the effect. Even when the applied field is set to about $0.1 \mathrm{~T}$, which would produce the maximum regions in the film at which the total field is small, much less than $1 \%$ of the film experiences a total field of less than $B_{\mathrm{HF}}$. Thus, the indirect mechanism of a fringe field added to an applied field causing hysteretic 


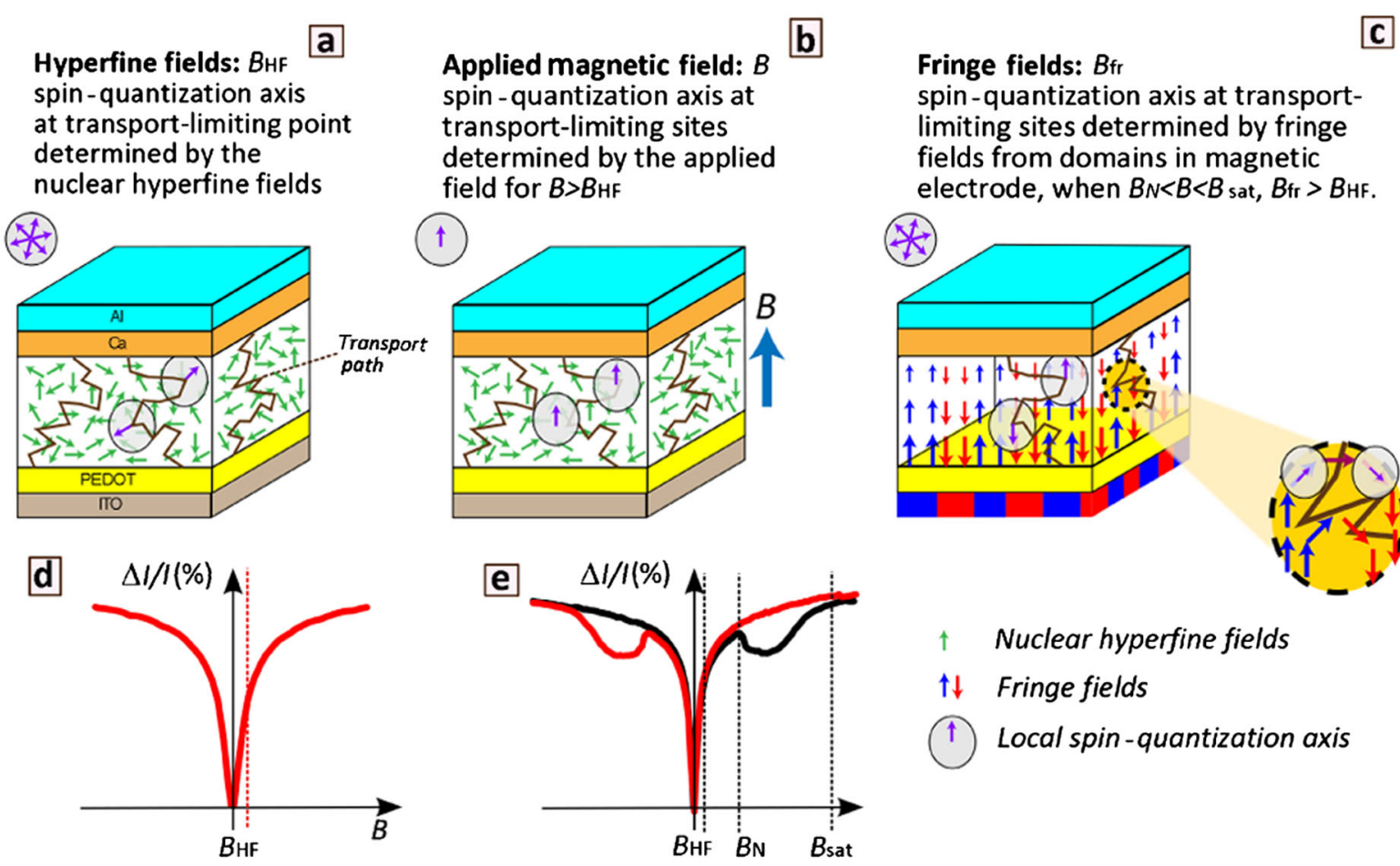

FIG. 7. Comparison of the magnetic fringe-field control of magnetoresistance with nonmagnetic organic magnetoresistance. (a) Transport along a hopping path (brown line) through the organic film is affected by variations in the local spin-quantization axis along the path. (The local spin-quantization axis shown by purple arrows.) (b) An applied magnetic field that exceeds the hyperfine field provides a uniform local spin-quantization axis in the film, enhancing transport. (c) The magnetic electrode produces inhomogeneous local fields that define a local spin-quantization axis independently of the nuclear-hyperfine field. (d) Magnetoresistance for a nonmagnetic organic device, showing a low-field regime and a high-field regime. (e) Magnetoresistance for the semi-spin valve, showing in addition an intermediate field behavior.

fringe-field effects through the nuclear-hyperfine mechanism, which was suggested to explain the small effects seen in Ref. [23], fails to explain our data. This conclusion is discussed in more detail in Appendix B.

\section{B. Mechanism by which the fringe field replaces an inhomogeneous hyperfine field}

We propose, instead, that the magnetic fringe fields provide a new, inhomogeneous local field that defines a local spin-quantization axis independently of the nuclearhyperfine field, as shown in Fig. 7(c). Specifically, the domains are present for magnetic fields greater than the domain-generation field $B_{\mathrm{N}}$ and less than the saturation field $B_{\text {sat }}$. (For a perpendicularly saturated ferromagnetic layer, no magnetic fringe fields are present in the absence of domains.) If $B_{\mathrm{N}}>B_{\mathrm{HF}}$, then a new region of modified resistivity occurs for magnetic fields between the domaingeneration field and the saturation field, causing the magnetoresistance to exhibit a feature similar to that at $B=0$, but now exhibited within a higher field range, as shown in Fig. 2 and schematically in Fig. 7(e).

The field-correlation lengths in Fig. 1 at different distances from the electrode can be correlated with the magnetoresistance measured in devices with different thicknesses of spacers (Fig. 2). Although controversy remains about the detailed mechanistic origin of the organic magnetoresistive effect with two nonmagnetic electrodes, most theories consider it a kinetic effect involving the spin-dependent recombination of two carriers or formation of two-carrier complexes. A simple model of the effect, which can be applied to many of these mechanisms $[10,11,14]$, analyzes the kinetics for two sites [30] that are considered to be bottlenecks in the carrier transport, one at most and the other at least singly occupied. This model as currently envisioned imagines these sites within a hopping length (about $0.1-1 \mathrm{~nm}$ ), for which the variation in fringe field is very small due to the large correlation lengths; the estimated effect on the conductivity from this two-site model is $\ll 1 \%$.

If we consider, within such a model, that the effect of the inhomogeneous field from the fringe fields of the magnetic layer acts in a fashion similar to the inhomogeneous hyperfine field, then a fundamental problem immediately emerges. The correlation length of the fringe field is too long to affect the resistance within a model similar to that used for nonmagnetic organic layers. We consider a model such as that put forward in Ref. [30], corresponding to a two-site model that can be simply solved. We assume a 


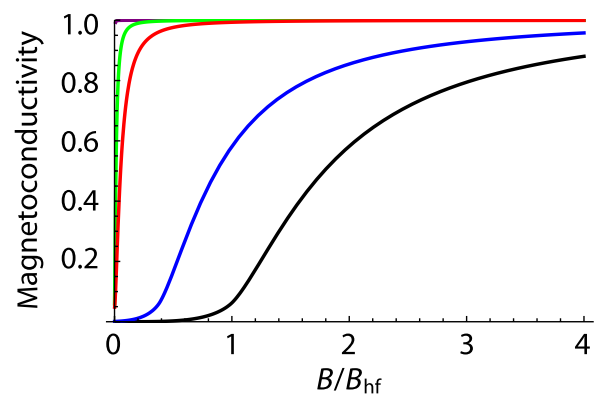

FIG. 8. Results of the two-site model for various correlation lengths of the inhomogeneous field: Organic magnetoconductivity, caused by fringe fields of differing correlation lengths, computed from the analytic function $g$ in Ref. [30]. Here, a magnetoconductivity of 1 corresponds to the high-field limit, and one of 0 corresponds to the zero-field magnetoconductivity for a spatially uncorrelated inhomogeneous field. The black curve is the result for an uncorrelated field, and the blue, red, green, and purple curves are for 2-, 5-, 10-, and 50-nm correlation lengths.

large value for the hopping length scale $(1 \mathrm{~nm})$, and calculate the magnetoconductivity curves when the inhomogeneous field is uncorrelated (the assumption used in Ref. [30]), or when it is much larger, as we have inferred in Fig. 1. The results are shown in Fig. 8. The effect goes away for longer correlation lengths within this model, and it should be unobservable at the correlation lengths characteristic of Fig. 1.

We thus find that inhomogeneous fields on length scales that are 2 orders of magnitude larger than typically involved in electronic transport in the organics have dramatic effects on the conductivity. A possible resolution might involve coordinated motion through a percolation cluster, whose radial size would be comparable to the thickness of the layer traversed $\left(30 \mathrm{~nm}\right.$ of $\left.\mathrm{Alq}_{3}\right)$. From Fig. 1, we see that the typical length scale of fringe-field correlation is about 50-100 nm, which ranges from comparable to much larger than the $\mathrm{Alq}_{3}$-layer thickness. For $\mathrm{Alq}_{3}$ layers located more than $50 \mathrm{~nm}$ from the magnetic electrode, the fringe-field correlation-length scale exceeds the typical size of the percolation cluster, and thus the fields acting on all sites within the cluster are typically parallel. In this situation, the spatially varying fringe field will not produce this magnetoresistive effect, as confirmed by Fig. 2. Further theoretical work is required to confirm this picture, for the current models of organic magnetoresistance fail to predict conductivity changes of this magnitude for inhomogeneous fields varying this slowly in space.

\section{CONCLUSIONS}

We have shown that the structure of a spatially varying magnetic fringe field originating from an unsaturated magnetic electrode dramatically modulates the magnetoresistance of an organic layer at room temperature, over the magnetic-field range where an applied field is comparable to the spatially varying fringe field. Fringe-field modulation of the nuclear-hyperfine organic-magnetoresistive effect fails to explain the size of the effect. The termination with $\mathrm{Pt}$ and use of a conductive PEDOT layer, and demonstration of similar results using devices with an electrically isolated ferromagnet and devices where PEDOT is replaced by ITO, rule out tunneling anisotropic magnetoresistance [31] as an explanation for our results. A novel model based on electrical transport in a spatially varying fringe field explains the approximate size of the effect, the hysteresis, and the dependence on distance from the ferromagnet that is generating the fringe fields.

Patterning of the domain structure of the magnetic electrode should permit a detailed engineering of the magnetoresistive curves caused by the single magnetic electrode, producing the greatest magnetic-field sensitivity in the field regime where domains are shifting in the magnetic electrode. Our results show that the sensitivity of the conductivity of an organic layer to the fringe field of a nearby magnetic film could be used to read the magnetic state of that film. As there would be no electrical connection between the magnetic film and the organic layer, this technique may have advantages if the magnetic film were nonconductive (e.g., were a magnetic insulator), or for a highly conductive magnetic film (avoiding an impedance mismatch). We also note that, despite the completely different physical origin of the effect, it is conceivable that curves such as those shown in Fig. 3(b) could be mistaken for those originating from actual spin valves. Thus, when measuring the magnetoresistance of a spin valve, it may be important to rule out the possibility that a fringe-field effect is influencing the data.

\section{ACKNOWLEDGMENTS}

This work was supported by ARO MURI Grant No. W911NF-08-1-0317 and NSF Grant No. ECS 0725280. F. M. acknowledges support from MC-IOF 253214. We acknowledge assistance from D. Arena, D. Backes, J.-M. Beaujour, D. Bedau, G. Chaves, S. Shen, P. Fischer, P. Manandhar, and M. Riemer. Device fabrication was partly carried out at the Cornell Nanofabrication Facility. X-ray measurements were performed at the Advanced Light Source at the Lawrence Berkeley National Laboratory.

\section{APPENDIX A: FURTHER CHARACTERIZATION OF THE MAGNETIC LAYER}

\section{Magneto-optic Kerr-effect measurements}

MOKE measurements were performed at room temperature using a HeNe laser. Changes in beam polarization were detected by measuring the intensity of light reflected off a sample located between two crossed polarizers as a function of the applied field. A silicon photodiode detector 


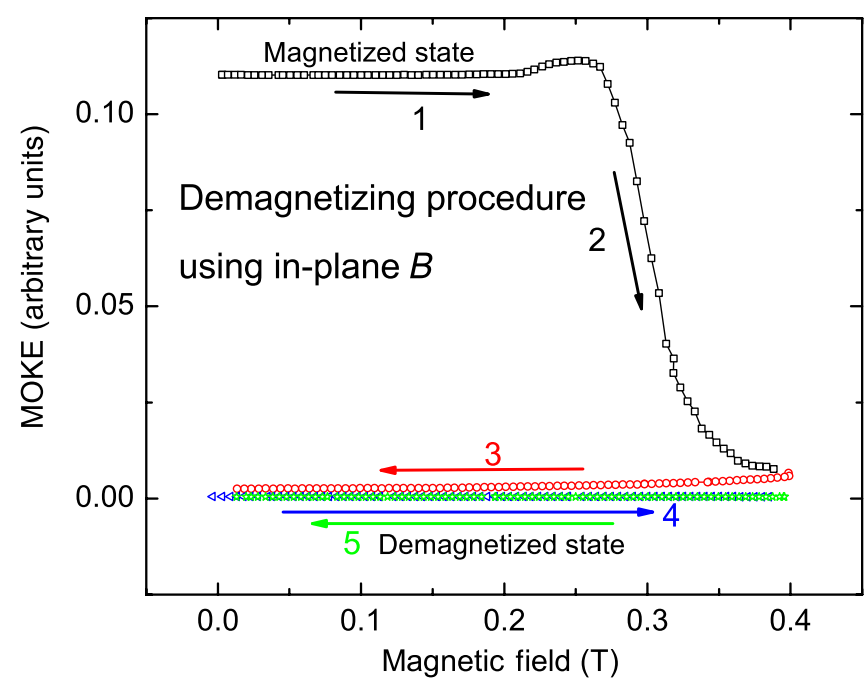

FIG. 9. Magnetization and demagnetization of a ferromagnetic electrode studied with polar MOKE technique. The sample starts in a magnetized state. To demagnetize the sample, we apply an increasing in-plane magnetic field (part 1 of the MOKE loop). Above a certain field strength, the sample becomes demagnetized, as evidenced by the abrupt decrease in MOKE signal (part 2 of the MOKE loop). Once demagnetized, the sample remains demagnetized (parts 3, 4, and 5). To remagnetize it, a perpendicular magnetic field must be applied.

and a lock-in amplifier (together with an optical chopper) were used to measure the light intensity.

\section{a. Magnetization and demagnetization procedure}

Here we describe the magnetizing and demagnetizing procedures that were used in the measurements of magnetoconductivity described in Sec. III. Since the easy axis of the magnetic electrode is perpendicular to the film plane, perpendicular applied fields can be used to magnetize the material. The relative perpendicular component of the magnetization of the material can be measured using MOKE. In-plane magnetic fields, on the other hand, can be used to demagnetize the material. Figure 9 shows MOKE measurements with an in-plane applied magnetic field. In this measurement, the sample start out in a magnetized state. To demagnetize the sample, we apply an increasing in-plane magnetic field (part 1 of the MOKE loop). Above a certain field strength, the sample becomes demagnetized in the perpendicular direction (i.e., the net magnetization along the $z$ direction vanishes, because the magnetization tends to align with the applied in-plane field), which is evidenced by the abrupt decrease of the MOKE signal (part 2 of the MOKE loop). When the field is removed (part 3 of the MOKE loop), magnetic moments return to the out-ofplane direction, forming up and down domains with a zero overall perpendicular magnetization. Continuing to apply in-plane magnetic fields again (parts 4 and 5 of the MOKE loop) tilts the magnetic moments in-plane, maintaining the perpendicular magnetization at zero (and the overall magnetization at zero when the applied field is removed). To magnetize the sample again in the perpendicular direction, an out-of plane magnetic field is required. Ramping a large out-of-plane magnetic field forth and back results in a partially magnetized state depending on the magnetic anisotropy of the sample and field-ramping range.

\section{Vibrating sample magnetometry}

VSM measurements are used to determine the magnetization versus an applied perpendicular field and the film's saturation magnetization, $M_{\mathrm{s}}$. Figure 10 shows that the hysteretic magnetoconductivity response of semi-spinvalves correlates well with the magnetic hysteresis loop of (unpatterned) bottom electrodes measured with VSM. The $M_{\mathrm{S}}$ values for the magnetic electrodes used in our studies were equal to approximately $7 \times 10^{5} \mathrm{~A} / \mathrm{m}$ [Fig. 10(a)] and approximately $5 \times 10^{5} \mathrm{~A} / \mathrm{m}$ [Fig. 10(b)].
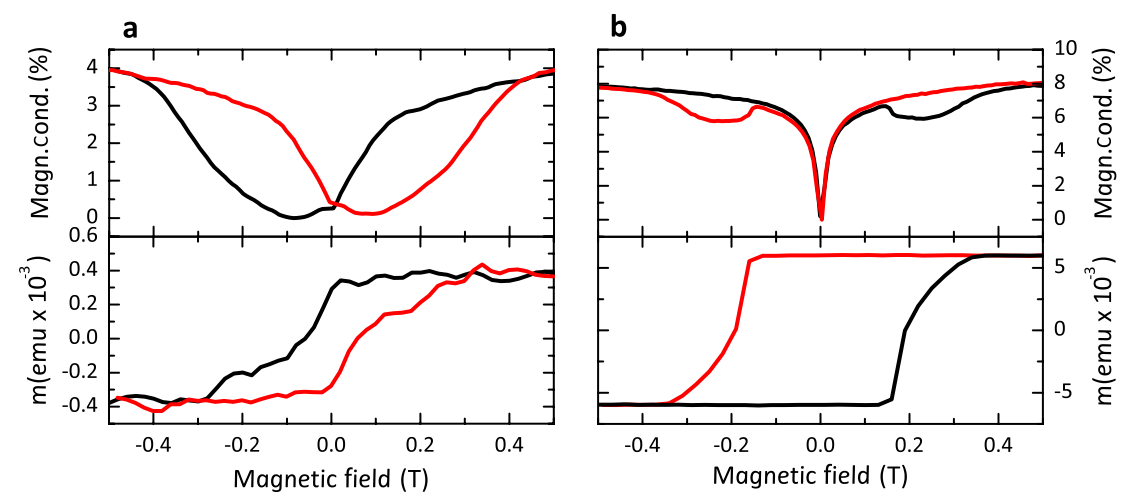

FIG. 10. Correlation between magnetoconductivity and magnetic hysteresis for different magnetic electrodes. The top panels of (a) and (b) show magnetoconductivity in completed semi-spin-valve devices; the bottom panels show VSM measurements of the magnetic electrodes before the device is completed (i.e., unpatterned bottom electrodes). The experiments were performed in a perpendicular applied magnetic field. The devices in the top panels of (a) and (b) use a 15-nm and 20-nm PEDOT spacer layer, respectively. 


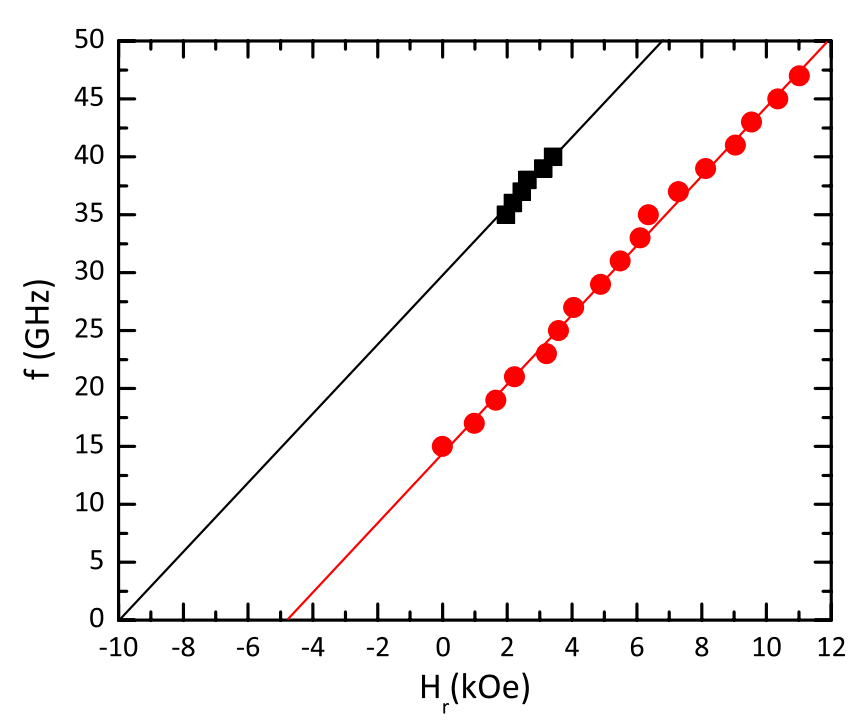

FIG. 11. Frequency dependence of the resonant field. The applied field is perpendicular to the film plane. The solid lines are fits using Eq. (1). The red line is from the electrode used in Figs. 3(a) and 3(c) and the black line is from the electrode in Figs. 3(b) and 3(d).

\section{Ferromagnetic resonance spectroscopy}

To determine the magnetic anisotropy of the magnetic electrodes, we have conducted FMR studies using a broadband technique. Films are placed on a coplanar waveguide, and frequencies from 4 to $45 \mathrm{GHz}$ are applied at room temperature. The transmitted signals are recorded while sweeping the magnetic field at constant microwave frequency $f$. (See Ref. [32] for more details on our FMR methods.) Here, we show results for two samples corresponding to the electrodes used in Fig. 3. Figure 11 shows the frequency dependence of the resonant field $H_{\mathrm{r}}$ with a field applied perpendicular to the film plane. The resonance condition is given by [33]

$$
f=\frac{\gamma}{2 \pi}\left(\mu_{0} H_{\mathrm{r}}-\mu_{0} M_{\mathrm{s}}+\frac{2 K}{M_{\mathrm{s}}}\right) .
$$

From the $f=0$ intercept of $f$ vs $H_{\mathrm{r}}$, the perpendicular anisotropy constant $K$ can be determined since we have measured $M_{\mathrm{s}}$ using VSM. If $\mu_{0} M_{\mathrm{s}}<2 K / M_{\mathrm{s}}$ (i.e., if $\left.H_{\mathrm{r}}(f=0)<0\right)$, the magnetic easy axis is perpendicular to the film plane, which is indeed the case for our samples. The anisotropies of the two films are $K \simeq 4.8 \times 10^{5} \mathrm{~J} / \mathrm{m}^{3}$ and $K \simeq 4.1 \times 10^{5} \mathrm{~J} / \mathrm{m}^{3}$, respectively. The slope of $f$ vs $H_{\mathrm{r}}$ is proportional to the gyromagnetic ratio $\gamma$, which is not appreciably different between the two samples, $\gamma \simeq 1.86 \times 10^{11} \mathrm{~Hz} / \mathrm{T}$ (i.e., $g \simeq 2.1$ ).

\section{Transmission $x-$ ray microscopy}

TXM combined with XMCD resolves magnetic signals of specific elements within a metallic structure with no need for having the element of interest either on the surface

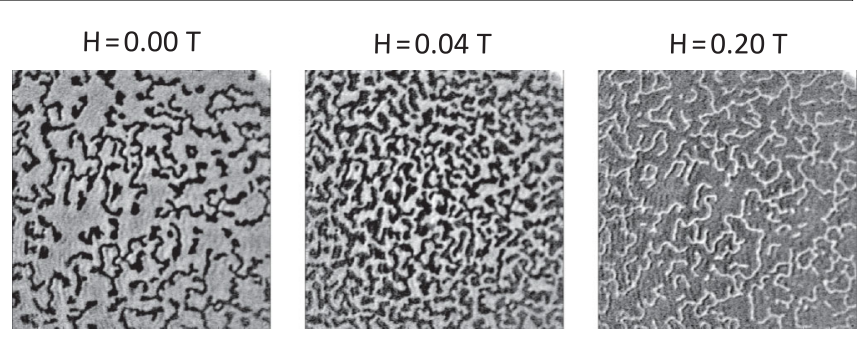

FIG. 12. X-ray microscopy images of a magnetic electrode at different applied perpendicular fields: 0, 0.04, and $0.2 \mathrm{~T}$. The images are selected from a magnetic-hysteresis loop shown in full in video 1 [28]. The image sizes are all $5 \times 5 \mu \mathrm{m}^{2}$.

or isolated. This is a photon-in/photon-out technique that does not perturb the sample during measurement, as compared to magnetic-force microscopy, and it is also insensitive to external fields applied to the sample. We have used this method to obtain images and movies of the magnetic domains as a function of applied field to correlate the macroscopic magnetization measurements that we have conducted by VSM and MOKE with microscopic magnetic-domain dynamics.

We have grown samples with the same composition we have used for the magnetic electrodes on silicon-supported $\mathrm{Si}_{3} \mathrm{~N}_{4}$-membrane substrates. The magnetic contrast in the images is obtained from the resonant magnetic term in the atomic-scattering factor that is first order in the magnetization. X-ray measurements were performed at the Advanced Light Source at Lawrence Berkeley National Laboratory. Images were taken with an XM-1 zone-plate microscope at beam line 6.1.2. The contrast with $\mathrm{x}$-ray energy tuned to the Co $L_{3}$ absorption edge (about $778 \mathrm{eV}$ and about $1.59 \mathrm{~nm}$ ) was measured. Imaging was performed with a two-dimensional CCD camera using elliptical polarization from above the synchrotron orbit plane in applied fields up to $0.3 \mathrm{~T}$. As the applied magnetic field is swept, existing domains grow and new ones appear. (See Fig. 12.) Video 1 [28] shows a full hysteresis loop from $-0.3 \mathrm{~T}$ to $0.3 \mathrm{~T}$. Video 2 [28] shows a hysteresis loop that starts from a zero-applied-field virgin state, a state that is achieved only after the material is deposited (and before any magnetic field has been applied to the sample.) Figure 12 shows three snapshots at different applied fields taken from video 1 .

\section{APPENDIX B: PREDICTIONS OF THE UNCORRELATED $B$-FIELD MODEL}

Here, we show that a model which assumes that the organic semiconductor responds just to the local $B$ fields and does not consider the field correlations fails to explain our experimental results.

The $B$ field in the organic layer is the sum of the applied field and the field due to the magnetic electrode. First, we note that the average magnetic field due to the contact, which is a very thin magnetic film, is always vanishing 


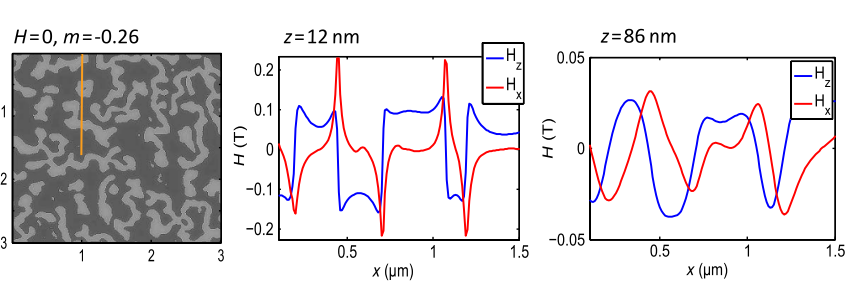

FIG. 13. Left: XMCD image of a magnetic electrode showing a magnetic configuration that corresponds to $H \approx 0$ and $M \approx-0.26 M_{\mathrm{s}}$ of Fig. 3(c). The middle and right panels show the fringe fields $\left(H_{x}\right.$ and $\left.H_{z}\right)$ along the XMCD image's vertical orange line at distances $z=12,86 \mathrm{~nm}$ from the surface of the magnetic electrode, respectively.

small, essentially zero, independent of the contact magnetization. This finding is associated with the fact that domains are magnetized up and down perpendicular to the film surface. Field lines originating from the top of the magnetic film must connect back to the top of the film. (The film is millimeters wide, so field lines going all the way around the lateral edges of the film are energetically unfavorable; only those lines very close to the film edges take this route.) Any field line that comes from the top of the film and returns to the top of the film does not contribute a net, or average, fringe field. Hence, the average of the fringe fields plus the applied fields is always just the applied field. The total (average) field in the organic layer is then just the externally applied magnetic field. We also note that a fully magnetized electrode produces no magnetic fringe fields.

Although the average fringe field is zero, its distribution and variance depend both on the distance from the magnetic electrode and on the magnetic-domain configuration. To understand the fringe-field distributions for different contact magnetizations and at different electrode distances, we first show the fringe fields for a given magnetization configuration. Figure 13 shows the domain configuration and the fringe fields $\left(H_{x}\right.$ and $\left.H_{z}\right)$ along a vertical line (it corresponds to the $x$ direction) at two different distances from the electrode. The magnetic configuration corresponds to $H \approx 0$ and $M \approx-0.26 M_{\mathrm{s}}$, as is the case in Fig. 3(c). The images have been taken with XMCD microscopy, and the fringe fields have been calculated with OOMMF code [26].

To see how the fringe-field distribution changes with the magnetic-domain states, we plot the fringe-field distribution, $H_{z}$ for the following three magnetic states: state 1 at $H \approx 0$ and with partial negative magnetization $M \approx$ $-0.26 M_{\mathrm{s}}$; state 2 at $H \approx 0.03$ and with a nearly zero magnetization $M \approx 0$; and state 3 at $H \approx 0.11 \mathrm{~T}$ with a
State 1: $H=0, m=-0.26$
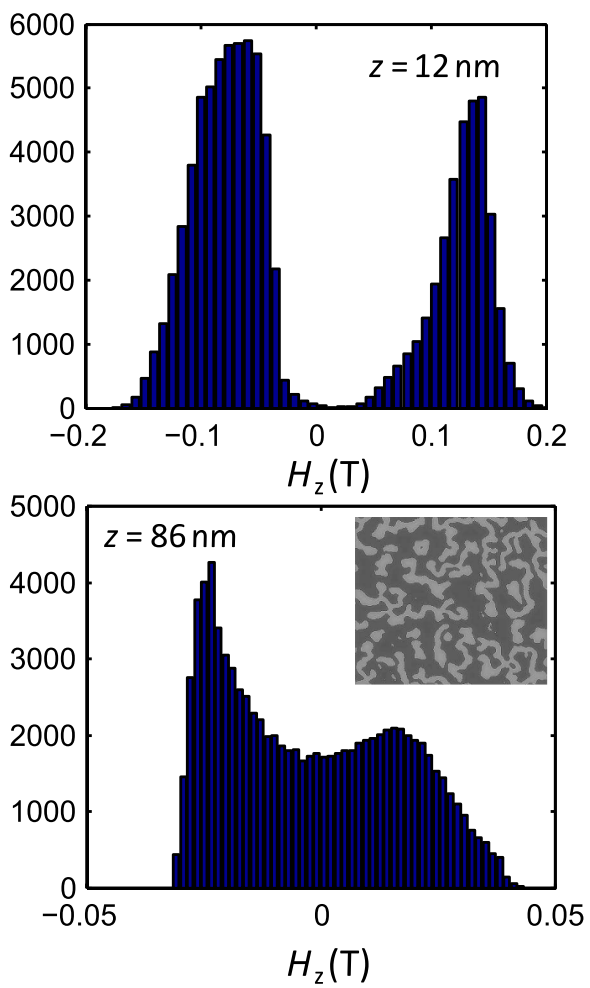

State 2: $H=0.03 \mathrm{~T}, m=0$
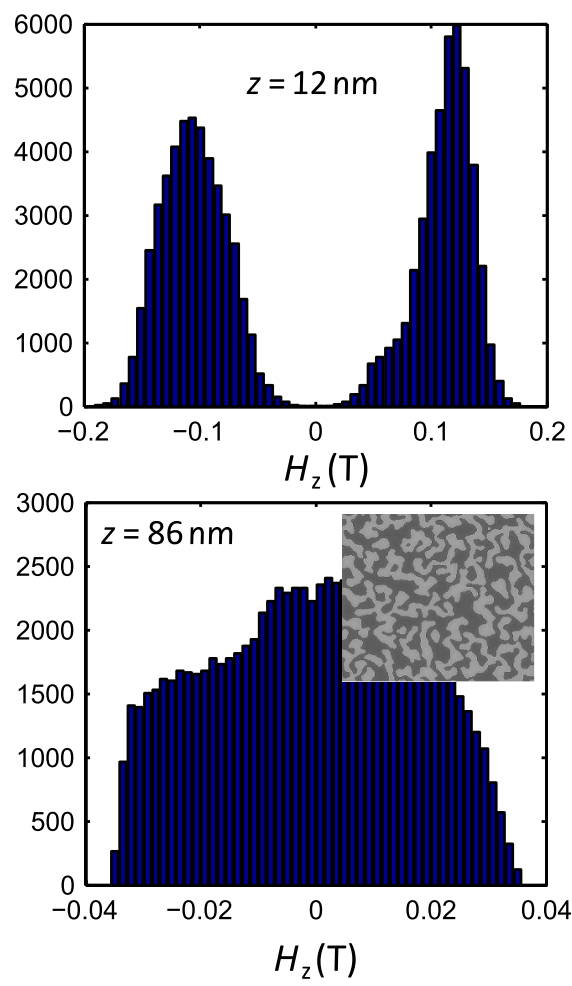

State 3: $H=0.11 \mathrm{~T}, m=0.26$
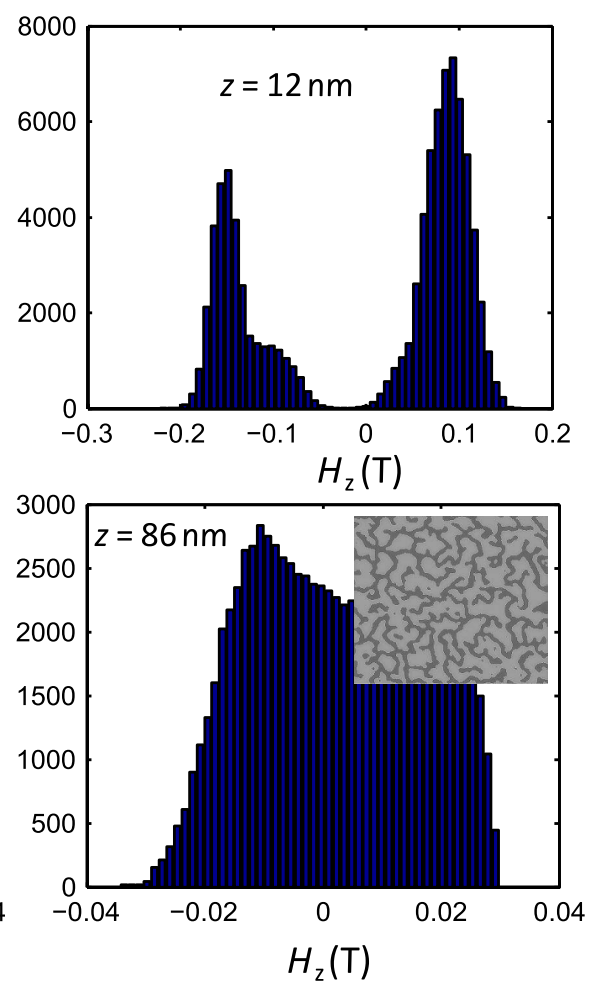

FIG. 14. Histograms (probability distribution) of the fringe fields in the $z$ direction $H_{z}$ from a magnetic electrode. Three states are shown; the values for $\mathrm{H}$ and $\mathrm{m}$ are approximate. The histograms correspond to magnetic fields at (row 1) $12 \mathrm{~nm}$ and (row 2) $86 \mathrm{~nm}$ from the ferromagnetic electrode, respectively. The grey insets are XMCD images of the magnetic domain structure which are $3 \times 3 \mu \mathrm{m}^{2}$. 

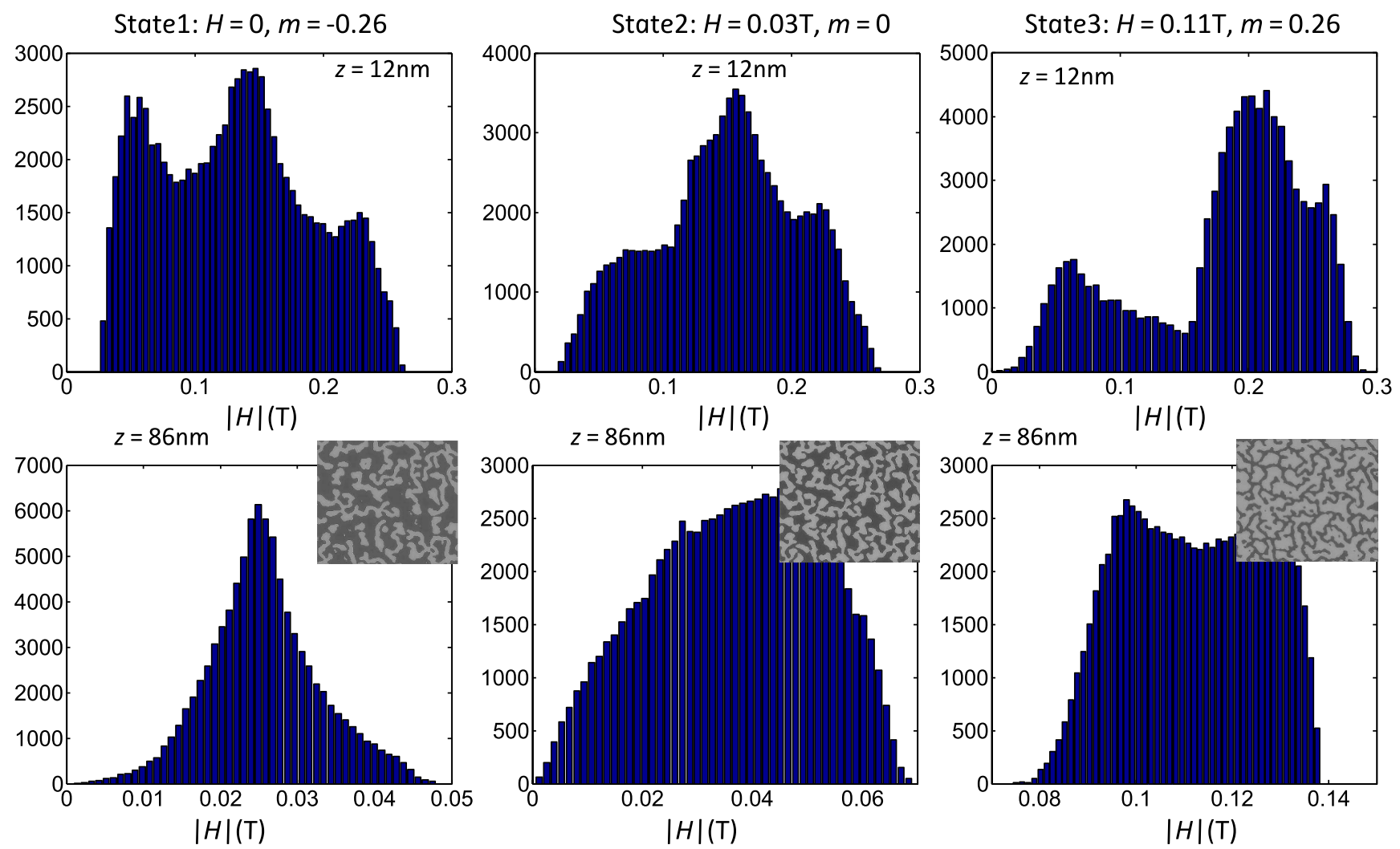

FIG. 15. Histograms (probability distribution) of the fringe fields plus the externally applied field, $|H|$, from a magnetic electrode. Three states are shown; the values for $\mathrm{H}$ and $\mathrm{m}$ are approximate. The histograms correspond to magnetic fields at (row 1) $12 \mathrm{~nm}$ and (row 2) $86 \mathrm{~nm}$ from the ferromagnetic electrode, respectively. The grey insets are XMCD images of the magnetic domain structure which are $3 \times 3 \mu \mathrm{m}^{2}$.

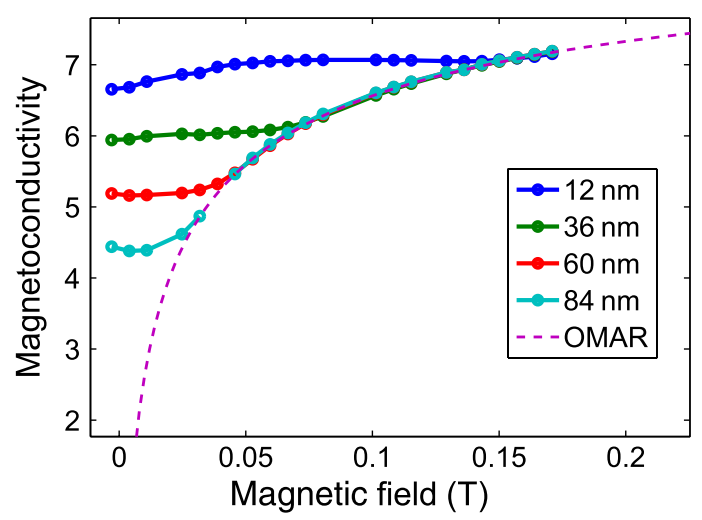

FIG. 16. Results of the uncorrelated $B$-field model. Computed organic magnetoconductivity caused by fringe fields of a magnetic electrode at different distances from the organic semiconductor. The magnetic fringe fields are determined from XMCD domain images of the magnetic electrodes. A large negative magnetic field first saturated the electrode. The computed curves correspond to the positive magnetic-field sweep of the black magnetization curve in Fig. 3. The purple dashed curve shows the OMAR response with a nonmagnetic electrode. positive magnetization $M \approx 0.26 M_{\mathrm{s}}$. (The external field $H_{0}$ is applied in the perpendicular $z$ direction.) The distributions shown in Fig. 14 correspond to the $z$ components of the fringe fields, $H_{z}$, and do not include the externally applied field (which would shift them uniformly).

\section{The uncorrelated $B$-field model}

We now consider a model in which the organic semiconductor's conductivity is a function of the local field that it experiences. Since OMAR is a scalar effect, the OMAR conductivity depends on the field magnitude, but not on its direction. Thus, the in-plane components of the fringe fields $H_{x}$ and $H_{y}$ need to be included to consider the organic's conductivity change. In Fig. 15, we plot the distributions of the total magnetic field at a fixed distance from the electrode $|H|$, which include all components of the fringe fields and the externally applied field. We see that, close to the magnetic electrode, the magnitude of the field is always considerable, on the order of $0.1 \mathrm{~T}$. That is, the fringe field from the magnetic electrode does not screen or compensate for the applied field.

To be quantitative, we consider a model in which the conductance in the organic material is due to channels in parallel, and the conductance of a channel is $P(H) C(H)$, 
where $P$ is the fringe-field probability distribution and $C(H)$ is the conductivity of a channel for a given $H=$ $h+H_{0}$. ( $h$ are the fringe fields, and $H_{0}$ is the externally applied field.) The net conductance is then just the sum of the conductances of each channel weighted by the number of such channels [i.e., $P(H)$ ]. In zero applied field, $H_{0}=0 \mathrm{~T}$ and $H(r)$ is due entirely to the fringe fields. At nonzero field, $H_{0} \neq 0$, one must include the applied field. The conductivity $C(H)$ is that of an OMAR device without a magnetic electrode. Figure 16 shows that, in a model in which the conductivity of the organic semiconductor depends only on the local field that the semiconductor experiences, there is a significant reduction of the magnetoconductance effect due to fringe fields. The closer the organic layer is to the magnetic electrode, the larger the fringe fields and the smaller the OMAR effect. Notice that the effect is precisely the opposite of what we observe in our experiments. One of the main reasons for this is that, close to the electrode, there are nearly no regions of the organic semiconductor with zero (or very small) magnetic field. (When $h_{z}=0$, then either $h_{x} \neq 0$ or $h_{y} \neq 0$.)

To summarize:

(1) The average fringe field from the magnetic electrode is zero--There is no resulting field from a magnetic electrode, and thus the magnetic electrode's field cannot compensate or screen the applied magnetic field. When the electrode is fully magnetized, there are no fringe fields.

(2) Magnetic fringe-field magnitudes are set by the material magnetization density and are large (approximately $0.1 \mathrm{~T}$ ) compared to the field scale of the hyperfine OMAR (approximately 0.01 T). The variance of the fringe-field distribution decays with the distance from the electrode, and a local conductivity model predicts an increase in the fringe-field OMAR with increasing electrode distance, precisely the opposite behavior to what is observed.

(3) The experiment shows a minimum in the magnetoconductivity at the electrode coercive fields, whereas a local conductivity model predicts that the minimum would occur at zero applied field.

[1] S. A. Wolf, D. D. Awschalom, R. A. Buhrman, J. M. Daughton, S. von Molnár, M.L. Roukes, A. Y. Chtchelkanova, and D. M. Treger, Spintronics: A SpinBased Electronics Vision for the Future, Science 294, 1488 (2001).

[2] D.D. Awschalom and M.E. Flatté, Challenges for Semiconductor Spintronics, Nature Phys. 3, 153 (2007).

[3] M.E. Flatté, Spintronics, IEEE Trans. Electron Devices 54, 907 (2007).

[4] M. N. Baibich, J. M. Broto, A. Fert, F. Nguyen Van Dau, F. Petroff, P. Etienne, G. Creuzet, A. Friederich, and
J. Chazelas, Giant Magnetoresistance of (001)Fe/(001)Cr Magnetic Superlattices, Phys. Rev. Lett. 61, 2472 (1988).

[5] G. Binasch, P. Grünberg, F. Saurenbach, and W. Zinn, Enhanced Magnetoresistance in Layered Magnetic Structures with Antiferromagnetic Interlayer Exchange, Phys. Rev. B 39, 4828 (1989).

[6] M. Johnson and R. H. Silsbee, Spin-Injection Experiment, Phys. Rev. B 37, 5326 (1988).

[7] C. Gould, C. Rüster, T. Jungwirth, E. Girgis, G. M. Schott, R. Giraud, K. Brunner, G. Schmidt, and L. W. Molenkamp, Tunneling Anisotropic Magnetoresistance: A Spin-Valvelike Tunnel Magnetoresistance Using a Single Magnetic Layer, Phys. Rev. Lett. 93, 117203 (2004).

[8] J. Kalinowski, M. Cocchi, D. Virgili, P. Di Marco, and V. Fattori, Magnetic Field Effects on Emission and Current in $\mathrm{Alq}_{3}$-Based Electroluminescent Diodes, Chem. Phys. Lett. 380, 710 (2003).

[9] T.L. Francis, Ö. Mermer, G. Veeraraghavan, and M. Wohlgenannt, Large Magnetoresistance at Room Temperature in Semiconducting Polymer Sandwich Devices, New J. Phys. 6, 185 (2004).

[10] V. N. Prigodin, J.D. Bergeson, D. M. Lincoln, and A.J. Epstein, Anomalous Room Temperature Magnetoresistance in Organic Semiconductor, Synth. Met. 156, 757 (2006).

[11] P. Desai, P. Shakya, T. Kreouzis, and W. P. Gillin, Magnetoresistance in Organic Light-Emitting Diode Structures under Illumination, Phys. Rev. B 76, 235202 (2007).

[12] B. $\mathrm{Hu}$ and $\mathrm{Y}$. Wu, Tuning Magnetoresistance between Positive and Negative Values in Organic Semiconductors, Nature Mater. 6, 985 (2007).

[13] F.L. Bloom, W. Wagemans, M. Kemerink, and B. Koopmans, Separating Positive and Negative Magnetoresistance in Organic Semiconductors, Phys. Rev. Lett. 99, 257201 (2007).

[14] P. A. Bobbert, T.D. Nguyen, F. W. A. van Oost, B. Koopmans, and M. Wohlgenannt, Bipolaron Mechanism for Organic Magnetoresistance, Phys. Rev. Lett. 99, 216801 (2007).

[15] J. D. Bergeson, V. N. Prigodin, D. M. Lincoln, and A. J. Epstein, Inversion of Magnetoresistance in Organic Semiconductors, Phys. Rev. Lett. 100, 067201 (2008).

[16] A. J. Drew, J. Hoppler, L. Schulz, F. L. Pratt, P. Desai, P. Shakya, T. Kreouzis, W. P. Gillin, A. Suter, N. A. Morley et al., Direct Measurement of the Electronic Spin Diffusion Length in a Fully Functional Organic Spin Valve by LowEnergy Muon Spin Rotation, Nature Mater. 8, 109 (2008).

[17] L. Schulz L. Nuccio, M. Willis, P. Desai, P. Shakya, T. Kreouzis, V. K. Malik, C. Bernhard, F. L. Pratt, N. A. Morley et al., Engineering Spin Propagation across a Hybrid Organic/Inorganic Interface Using a Polar Layer, Nature Mater. 10, 39 (2010); ,Engineering Spin Propagation across a Hybrid Organic/Inorganic Interface Using a Polar Layer, 10, 252 (2011).

[18] V. A. Dediu, L. E. Hueso, I. Bergenti, and C. Taliani, Spin Routes in Organic Semiconductors, Nature Mater. 8, 707 (2009); Spin Routes in Organic Semiconductors, 8, 850(E) (2009).

[19] V. Dediu, M. Murgia, F. C. Matacotta, C. Taliani, and S. Barbanera, Room Temperature Spin Polarized 
Injection in Organic Semiconductor, Solid State Commun. 122, 181 (2002).

[20] Z.H. Xiong, D. Wu, Z. V. Vardeny, and J. Shi, Giant Magnetoresistance in Organic Spin-Valves, Nature (London) 427, 821 (2004).

[21] Sayani Majumdar, R. Laiho, P. Laukkanen, I. J. Väyrynen, Himadri S. Majumdar, and R. Österbacka, Application of Regioregular Polythiophene in Spintronic Devices: Effect of Interface, Appl. Phys. Lett. 89, 122114 (2006).

[22] J.-W. Yoo, C.-Y. Chen, H. W. Jang, C. W. Bark, V. N. Prigodin, C. B. Eom, and A. J. Epstein, Spin Injection/ Detection Using an Organic-Based Magnetic Semiconductor, Nature Mater. 9, 638 (2010); Spin Injection/ Detection Using an Organic-Based Magnetic Semiconductor, 9, 778E (2010).

[23] G. Salis, S. F. Alvarado, M. Tschudy, T. Brunschwiler, and R. Allenspach, Hysteretic Electroluminescence in Organic Light-Emitting Diodes for Spin Injection, Phys. Rev. B 70, 085203 (2004).

[24] O. Hellwig, A. Berger, J. B. Kortright, and E. E. Fullerton, Domain Structure and Magnetization Reversal of Antiferromagnetically Coupled Perpendicular Anisotropy Films, J. Magn. Magn. Mater. 319, 13 (2007).

[25] A. D. Kent, J. Yu, U. Ruediger, and S. S. P. Parkin, Domain Wall Resistivity in Epitaxial Ferromagnetic Thin Film Microstructures, J. Phys. Condens. Matter 13, R461 (2001).

[26] M. J. Donahue and D. G. Porter, Interagency Report No. NISTIR 6376, National Institute of Standards and Technology, Gaithersburg, MD (1999) [http://math.nist .gov/oommf/].

[27] P. Fischer, T. Eimüller, G. Schütz, G. Denbeaux, A. Pearson, L. Johnson, D. Attwood, S. Tsunashima, M. Kumazawa, N. Takagi, M. Köhler, and G. Bayreuther,
Element-Specific Imaging of Magnetic Domains at $25 \mathrm{~nm}$ Spatial Resolution Using Soft X-Ray Microscopy, Rev. Sci. Instrum. 72, 2322 (2001).

[28] See Supplemental Material at http://link.aps.org/ supplemental/10.1103/PhysRevX.2.021013 for videos of the magnetization dynamics. The XMCD videos of hysteresis loops of magnetic electrodes are shown with the field applied perpendicular to the samples. Video 1 shows a magnetization loop from -0.3 to 0.3 Tesla. Video 2 corresponds to a virgin sample; the magnetization loop goes from zero applied field to 0.3 Tesla and back to -0.3 Tesla.

[29] Ö. Mermer, G. Veeraraghavan, T. L. Francis, Y. Sheng, D. T. Nguyen, M. Wohlgenannt, A. Köhler, M. K. Al-Suti, and M. S. Khan, Large Magnetoresistance in Nonmagnetic $\pi$-Conjugated Semiconductor Thin Film Devices, Phys. Rev. B 72, 205202 (2005).

[30] W. Wagemans, F.L. Bloom, P.A. Bobbert, M. Wohlgenannt, and B. Koopmans, A Two-Site Bipolaron Model for Organic Magnetoresistance, J. Appl. Phys. 103, 07F303 (2008).

[31] M. Grünewald, M. Wahler, F. Schumann, M. Michelfeit, C. Gould, R. Schmidt, F. Würthner, G. Schmidt, and L. W. Molenkamp, Tunneling Anisotropic Magnetoresistance in Organic Spin Valves, Phys. Rev. B 84, 125208 (2011).

[32] J.-M. L. Beaujour, W. Chen, K. Krycka, C.-C. Kao, J.Z. Sun, and A. D. Kent, Ferromagnetic Resonance Study of Sputtered Co|Ni Multilayers, Eur. Phys. J. B 59, 475 (2007).

[33] J.-M. Beaujour, D. Ravelosona, I. Tudosa, E. E. Fullerton, and A.D. Kent, Ferromagnetic Resonance Linewidth in Ultrathin Films with Perpendicular Magnetic Anisotropy, Phys. Rev. B 80, 180415(R) (2009). 